Revista Científica do Corpo de Bombeiros Militar de Pernambuco

XVIII Seminário Nacional de Bombeiros - Foz do Iguaçu PR

Vol.04 No11 - Edição Especial XVIII SENABOM - ISSN 2359-4829

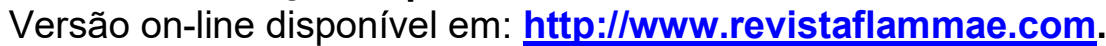

\title{
FATORES DE RISCO PARA DOENÇAS CARDIOVASCULARES ENTRE BOMBEIROS MILITARES SUBMETIDOS À INSPEÇÃO DE SAÚDE PERIÓDICA
}

\author{
CARDIOVASCULAR DISEASE RISK FACTORS IN FIREFIGHTERS SUBMITTED TO \\ PERIODIC HEALTH EXAMINATION
}

\author{
Mariana Tschoepke Aires ${ }^{l}$ \\ Roberto Kazumi Baldas Miura \\ Melanie Hurel Barroso
}

\section{Resumo}

O presente estudo é seccional, descritivo sobre a prevalência de fatores de risco para doenças cardiovasculares (DCV) em Bombeiros Militares (BM). O objetivo é determinar a prevalência dos principais fatores de risco para DCV entre BM. Foram analisados os dados das inspeções de saúde periódicas realizadas em 2015. Foram estudados $800 \mathrm{BM}$ (idade média 40,5 $\pm 7,5$ anos), $85,6 \%$ sexo masculino. A prevalência de sobrepeso/obesidade foi de $70,9 \%$, hipercolesterolemia $45 \%$, hipertensão arterial $17,4 \%$, diabetes mellitus $33,5 \%$ e sedentarismo $35,5 \%$. Em conclusão, alta proporção de BM neste estudo apresentam fatores de risco modificáveis para DCV. Estes achados sugerem que os BM podem se beneficiar de iniciativas de adoção de estilos de vida saudáveis.

Palavras-chave: Doenças Cardiovasculares. Dislipidemias. Obesidade. Hipertensão. Fatores de risco.

\begin{abstract}
This is a cross-sectional descriptive study on the prevalence of risk factors for cardiovascular disease (CVD) among the military firefighters (MF). The objective is to examine the prevalence of the main CVD risk factors among firefighters. Data from their periodic health inspection during 2015 were analyzed. We studied $800 \mathrm{MF}$, (mean age 40,5 $\pm 7,5$ years), 85,6\% male. The prevalence of obesity/overweight was 70,9\%, hypercholesterolemia $45 \%$, arterial hypertension $17,4 \%$, diabetes mellitus 3,5\%, physical inactivity 35,5\%. In conclusion, a high proportion of MF in this study presented modifiable risk factors for CVD. These findings suggest that they would benefit from healthy lifestyle initiatives.
\end{abstract}

Key words: Cardiovascular Diseases. Dyslipidemias. Obesity. Hypertension. Risk factors

1 Corpo de Bombeiros Militar do Estado do Rio de Janeiro, Doutora em Saúde Coletiva, e-mail: saude.cbmerj@gmail.com 


\section{INTRODUÇÃO}

De acordo com a Organização Mundial da Saúde, aproximadamente 17 milhões de pessoas morrem todos os anos em decorrência de doenças cardiovasculares (DCV) e dentre estas, 3 milhões de mortes poderiam ser evitadas (MENDIS et al, 2011). No Brasil as Doenças Crônicas Não-transmissíveis, entre estas as DCV, são as principais causas de morte, e seu controle vem ganhando atenção nas agendas públicas de promoção de saúde. Ações devem ser implementadas para o controle dos fatores de risco para o desenvolvimento destas, com ênfase para a obesidade, hipertensão, hipercolesterolemia e diabetes (REZENDE et al, 2006 e SCHMIDT et al, 2011).

As doenças cardiovasculares são a principal causa de morte entre bombeiros norte-americanos em atividade, sendo responsáveis por 45\% dos óbitos (KALES, 2007). A prevalência de obesidade entre bombeiros norte-americanos vem aumentando nos últimos anos, e associa-se a aumento da frequência de hipercolesterolemia e hipertensão, potencializando o risco de eventos cardiovasculares (POSTON, 2011). Bombeiros voluntários ou não nos Estados Unidos apresentam altas prevalências de obesidade e sobrepeso, chegando a $82,5 \%$, valores superiores àqueles encontrados na população geral (SOTERIADES, 2005).

A aptidão cardiorrespiratória foi menor em militares obesos quando comparados àqueles com peso ideal (NOGUEIRA, 2016 e OLIVEIRA, 2008) . Entre Policiais Militares (PM) no Brasil, a prevalência de sobrepeso e obesidade foi variou de 58 a $68 \%$ e a prevalência de hipertensão arterial sistêmica foi de 5,3\% e hipercolesterolemia 28\% (CALAMITA, 2010). No Brasil, a prevalência de sobrepesou variou de 27 a 54.3\% e obesidade variou de $14.7 \%$ a $18.5 \%$ entre Bombeiros Militares (BM) (SMITH, 2012). Neste grupo a aproximadamente $30 \%$ apresentavam dislipidemia (RODRIGUES, 2012). Observa-se que tanto BM quanto PM no nosso meio estão acima do peso (MINAYO et al, 2011).

Tais achados são alarmantes visto que os eventos cardiovasculares agudos que acometem os militares em serviço incidem mais naqueles com doenças de base. Além 


\section{Revista FLAMMAE}

Revista Científica do Corpo de Bombeiros Militar de Pernambuco

XVIII Seminário Nacional de Bombeiros - Foz do Iguaçu PR

Vol.04 No11 - Edição Especial XVIII SENABOM - ISSN 2359-4829

Versão on-line disponível em: http://www.revistaflammae.com.

disso, a eficácia das tarefas desenvolvidas pelos Bombeiros militares em operações de combate à incêndios, busca e salvamento, sejam terrestres, marítimas ou aéreas, depende de um bom condicionamento físico.

Revisão da literatura mostrou que há uma lacuna no conhecimento dos fatores de risco para doenças cardiovasculares entre BM. O objetivo deste trabalho foi identificar tais fatores em BM submetidos à inspeção de saúde periódica (ISP) na Corporação.

\section{DESENVOLVIMENTO}

Metodologia

Foi realizado um estudo seccional observacional descritivo envolvendo amostra de $800 \mathrm{BM}$ em atividade submetidos à ISP no período de janeiro a junho de 2015.

Todos os Bombeiros Militares em atividade são submetidos à ISP que consiste de anamnese e avaliações clínicas pelos médicos e aferição de dados antropométricos (peso, altura e circunferência abdominal) bem como pressão arterial e frequência cardíaca pelas praças auxiliares de enfermagem. Na anamnese foram indagadas a história pessoal de hipertensão arterial e diabetes, bem como história de tabagismo e prática de atividade física.

A pressão arterial (PA) foi aferida com o militar sentado e em repouso com esfigmomanômetro manual. Foi considerada hipertensão arterial sistêmica (HAS) níveis pressóricos acima de $140 \mathrm{mmHg}$ para pressão sistólica e ou de $90 \mathrm{mmHg}$ para pressão diastólica (SOCIEDADE BRASILEIRA DE HIPERTENSÃO, 2010). O peso foi aferido por praças previamente treinados para tal, utilizando-se uma balança mecânica com precisão de $0,1 \mathrm{~kg}$ e capacidade máxima de $150 \mathrm{~kg}$. A estatura foi mensurada com a utilização de um estadiômetro fixo, com precisão de $0,1 \mathrm{~cm}$ e extensão máxima de dois metros.

A partir destas variáveis, o índice de massa corporal (IMC) foi calculado e classificado como normal (IMC $>18,5$ e $<25 \mathrm{~kg} / \mathrm{m} 2$ ), sobrepeso (IMC $\geq 25$ e $<30$ $\mathrm{kg} / \mathrm{m} 2$ ) e obeso (IMC $\geq 30 \mathrm{~kg} / \mathrm{m} 2$ ), segundo as diretrizes da ABESO (2016) . A circunferência abdominal (CA) foi obtida na altura da cicatriz umbilical por fita 
Revista Científica do Corpo de Bombeiros Militar de Pernambuco

XVIII Seminário Nacional de Bombeiros - Foz do Iguaçu PR

Vol.04 N011 - Edição Especial XVIII SENABOM - ISSN 2359-4829

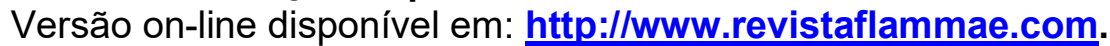

antropométrica maleável com precisão de $0,1 \mathrm{~cm}$. Em mulheres foi considerada aumentada CA maior que $80 \mathrm{~cm}$ e em homens CA maior que $94 \mathrm{~cm}$.

Para a definição dos pontos de corte referentes aos testes laboratoriais, foram utilizados os critérios da V Diretriz Brasileira de Dislipidemia e Prevenção da Aterosclerose da Sociedade Brasileira de Cardiologia (XAVIER, 2013): colesterol total Desejável $(<200 \mathrm{mg} / \mathrm{dL})$, limítrofe $(200$ a $239 \mathrm{mg} / \mathrm{dL})$ e alto $(\geq 240 \mathrm{mg} / \mathrm{dL})$ e triglicerídeos (na faixa desejável $(<150 \mathrm{mg} / \mathrm{dL})$, limítrofe $(150$ a $200 \mathrm{mg} / \mathrm{dL})$ e alta $(\geq$ $200 \mathrm{mg} / \mathrm{dL}$ )). Glicemia foi considerada alta acima de $100 \mathrm{mg} / \mathrm{dl}$ (de OLIVEIRA, 2015).

Os dados foram armazenados e analisados no software STATA versão 7.0, para descrição de frequências simples, cálculo de médias e desvio-padrão das variáveis qualitativas.

O presente projeto está em consonância com a Resolução 466 de 12/12/12 e suas complementares e foi aprovado pelo Comitê de Ética em Pesquisa do HOSPITAL NAVAL MARCÍLIO DIAS em 08/09/2015. Não houve suporte financeiro nem conflitos de interesse.

\section{Resultados}

Foram incluídos no estudo 800 Bombeiros Militares em atividade que fizeram Inspeção de Saúde Periódica no ano de 2015. A tabela 1 mostra os dados sóciodemográficos da população estudada. As Características da População estudada estão resumidas na Tabela 2.

Os BM apresentavam idade média de 40,5 \pm 7,5 anos, variando de 19 a 56 anos de idade. $85,6 \%$ dos militares estudados pertenciam ao sexo masculino, com média de idade 41,1 7,5 anos, variando de 19 a 56 anos de idade. No grupo feminino, havia 115 participantes, com idade média de 37,4 $\pm 5,8$ anos, variando de 25 a 53 anos de idade. 
Revista Científica do Corpo de Bombeiros Militar de Pernambuco

XVIII Seminário Nacional de Bombeiros - Foz do Iguaçu PR

Vol.04 No11 - Edição Especial XVIII SENABOM - ISSN 2359-4829

Versão on-line disponível em: http://www.revistaflammae.com.

Tabela 1. Dados sócio-demográficos dos BM ( $\mathrm{n}=800)$.

\begin{tabular}{|c|c|c|}
\hline Características & & Número (Percentual) \\
\hline \multirow{2}{*}{ Sexo } & Masculino & $685(85,63 \%)$ \\
\cline { 2 - 3 } & Feminino & $115(14,38 \%)$ \\
\hline \multirow{2}{*}{ Posto } & Oficial & $102(12,75 \%)$ \\
\cline { 2 - 3 } & Praça & $651(81,5 \%)$ \\
\cline { 2 - 3 } & Cadete & $46(5,75 \%)$ \\
\hline
\end{tabular}

Tabela 2. Características Gerais da População estudada.

\begin{tabular}{|c|c|}
\hline Idade (média \pm DP) & $40,5 \pm 7,5$ \\
\hline IMC (média \pm DP) & $27,1 \pm 3,9$ \\
\hline Glicemia (média \pm DP) & $91,7 \pm 20,4$ \\
\hline Pressão sistólica em repouso (média \pm DP) & $123,3 \pm 15,2$ \\
\hline Pressão diastólica em repouso (média \pm DP) & $81,1 \pm 18,7$ \\
\hline Colesterol total (média \pm DP) & $200,1 \pm 47,9$ \\
\hline Triglicerídeos total (média \pm DP) & $131,2 \pm 98,2$ \\
\hline Circunferência abdominal sexo masculino (média \pm DP) & $93,8 \pm 10,4$ \\
\hline Circunferência abdominal sexo feminino (média \pm DP) & $81,1 \pm 11,2$ \\
\hline
\end{tabular}

A tabela 3 mostra a avaliação antropométrica dos militares. Quando analisamos os sexos em separado, observamos que entre os homens a média do IMC é de 27,4 $\pm 3,7$, variando de 17,6 a 47,8 e entre as mulheres a média é de $24,6 \pm 3,8$, variando de 16,7 a 37,3. A circunferência abdominal estava aumentada em $44 \%$ dos militares, sendo que dentre os militares do sexo masculino, $45 \%$ apresentavam a circunferência aumentada e dentre as militares, 39\% tinham circunferência abdominal acima dos valores de 
Revista Científica do Corpo de Bombeiros Militar de Pernambuco

XVIII Seminário Nacional de Bombeiros - Foz do Iguaçu PR

Vol.04 No11 - Edição Especial XVIII SENABOM - ISSN 2359-4829

Versão on-line disponível em: http://www.revistaflammae.com.

referência. Obesidade (IMC $\geq 30 \mathrm{~kg} / \mathrm{m}^{2}$ ) foi observada em $8 \%$ e 21,7\% dos BM do sexo feminino e masculino, respectivamente.

Quanto à aferição da pressão arterial, $11 \%$ dos militares apresentavam hipertensão no momento do exame. Quando indagados sobre a história pessoal, 3,5\% $(\mathrm{n}=28)$ e 17,4\% ( $\mathrm{n}=139)$ dos BM referiram ser diabéticos e hipertensos em tratamento, respectivamente. A prevalência de HAS nos militares do sexo masculino e feminino foi $19,3 \%$ e 5,2\% respectivamente. Já a frequência de diabetes mellitus foi de $3,7 \%$ no sexo masculino e $\quad 2,6 \%$ no sexo feminino.

Quanto ao tabagismo, 7,0\% (n=56) referiram fumar ou já terem fumado em algum momento, sendo este percentual maior entre os homens (dentre os fumantes, 95\% pertenciam ao sexo masculino). 50,8 \% dos militares referiram praticar atividade física na corporação e $64,5 \%$ fora da corporação. Na tabela 4 estão descritos os resultados de exames laboratoriais dos BM.

Tabela 3. Avaliação antropométrica da população estudada $(\mathrm{n}=752)$.

\begin{tabular}{|c|c|}
\hline Avaliação antropométrica & Total $(\mathrm{n}=752)$ e percentual \\
\hline Baixo peso: IMC $<18,5 \mathrm{~kg} / \mathrm{m} 2$ & $5(0,6 \%)$ \\
\hline Normal: IMC $>18,5 \mathrm{e}<25 \mathrm{~kg} / \mathrm{m} 2$ & $216(28,5 \%)$ \\
\hline Sobrepeso: IMC $\geq 25 \mathrm{e}<30 \mathrm{~kg} / \mathrm{m} 2$ & $384(51,5 \%)$ \\
\hline Obeso I: IMC $\geq 30 \mathrm{e}<35 \mathrm{~kg} / \mathrm{m} 2$ & $113(15 \%)$ \\
\hline Obeso II: IMC $\geq 35 \mathrm{e}<40 \mathrm{~kg} / \mathrm{m} 2$ & $31(4 \%)$ \\
\hline Obeso III: IMC $\geq 40 \mathrm{~kg} / \mathrm{m} 2$ & $3(0,4 \%)$ \\
\hline
\end{tabular}

Tabela 4. Exames complementares dos BM.

\begin{tabular}{|c|c|}
\hline Exame & Total e percentual \\
\hline Colesterol total Desejável $<200 \mathrm{mg} / \mathrm{dL}$ & $497(55,1 \%)$ \\
\hline Colesterol total Limítrofe $200 \mathrm{a} 239 \mathrm{mg} / \mathrm{dL}$ & $229(28,9 \%)$ \\
\hline Colesterol total Alto $\geq 240 \mathrm{mg} / \mathrm{dL}$ & $127(16 \%)$ \\
\hline Triglicerídeos - Desejável $<150 \mathrm{mg} / \mathrm{dL}$ & $558(71,5 \%)$ \\
\hline
\end{tabular}


Revista Científica do Corpo de Bombeiros Militar de Pernambuco

XVIII Seminário Nacional de Bombeiros - Foz do Iguaçu PR

Vol.04 No11 - Edição Especial XVIII SENABOM - ISSN 2359-4829

Versão on-line disponível em: http://www.revistaflammae.com.

\begin{tabular}{|c|c|}
\hline Triglicerídeos - Limítrofe 150 a $200 \mathrm{mg} / \mathrm{dL}$ & $112(14,3 \%)$ \\
\hline Triglicerídeos - Alto $\geq 200$ a $499 \mathrm{mg} / \mathrm{dL}$ & $102(13 \%)$ \\
\hline Triglicerídeos - Muito alto: $\geq 500 \mathrm{mg} / \mathrm{dL}$ & $8(1 \%)$ \\
\hline Glicemia $>100 \mathrm{mg} / \mathrm{dL}$ & $120(15 \%)$ \\
\hline
\end{tabular}

\section{Discussão}

Este é o primeiro estudo a avaliar os fatores de risco para doenças cardiovasculares entre bombeiros militares em atividade no Estado. Dentre bombeiros norte-americanos, estas são as principais causas de morte durante o exercício da profissão (KALES, 2007).

Fatores como a atividade extenuante, obesidade, baixos índices de atividade física são apontados como causadores destas doenças. O percentual de gordura corporal associa-se com o risco aumentado de doença cardiovascular ateroesclerótica (LI et al, 2018, LI et al, 2017). Além de aumentar tal risco, obesidade e preparo físico inadequado podem interferir na resposta dos bombeiros em situações de emergência (SOTERIADES , 2001).

O presente estudo mostrou ainda maior frequência de tabagismo no sexo masculino, como relatado na literatura. Na população geral, $38,7 \%$ a 34,4\% dos indivíduos são sedentários (REZENDE et al, 2006), percentual semelhante ao observado no nosso estudo (35,5\%). Como o sedentarismo é fator de risco para a obesidade, além do fato de um bom condicionamento físico e capacidade cardiorrespiratória serem fundamentais para o desempenho da atividade de $\mathrm{BM}$, é mister promover programas de incentivo à prática de atividades físicas neste segmento ocupacional.

O risco de desenvolver doenças cardiovasculares é maior entre bombeiros do que na população geral, como demonstrado em estudo de coorte conduzido na Dinamarca (PEDERSEN et al, 2018). Além disso, aproximadamente 10\% dos bombeiros apresentam síndrome metabólica, caracterizada por diabetes melitus, dislipidemia (hipertrigliceridemia e lipoproteína de alta densidade -HDL- diminuída) e hipertensão arterial sistêmica (LI et al, 2017). Na população geral observa-se maior 
Revista Científica do Corpo de Bombeiros Militar de Pernambuco

XVIII Seminário Nacional de Bombeiros - Foz do Iguaçu PR

Vol.04 No11 - Edição Especial XVIII SENABOM - ISSN 2359-4829

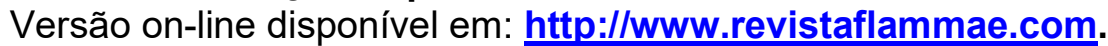

frequência de síndrome metabólica entre indivíduos com grupo sobrepeso e obesidade, principalmente no sexo masculino.

Entre os PM em São Paulo, a prevalência de sobrepeso e dislipidemia foi de 54\%, significativamente maior no sexo masculino (BARBOSA e SILVA, 2013). Entre os BM também observou-se maior prevalência destes agravos no sexo masculino.

$\mathrm{Na}$ Bélgica estudo avaliando o desempenho cardiorespiratório de bombeiros mostrou que o valor médio do índice de massa corporal (IMC) foi de $26,0 \mathrm{~kg} / \mathrm{m} 2$ (desvio padrão de 3,8; variando de 17,5 a 42). Aproximadamente $52 \%$ dos bombeiros neste país apresentavam sobrepeso ou obesidade (KISS et al, 2014).

Os resultados são muito semelhantes aos observados no nosso meio, em que 51,6\% dos nossos militares apresentam sobrepeso e aproximadamente $20 \%$ apresentam obesidade. No nosso meio, entretanto, o valor médio do IMC foi mais alto - 27,1 $\mathrm{kg} / \mathrm{m} 2$. Não obstante considerar-se a massa muscular nestes valores, a média de IMC no nosso meio situa-se no intervalo de classificação de sobrepeso, o que sugere intervenção imediata.

No Canadá foram realizados estudos avaliando a saúde de bombeiros comparandose os sexos (TRUDEAU et al, 2018). Entre os homens observou-se prevalência de obesidade de $23,6 \%$, e no nosso meio a prevalência foi de $19,2 \%$. Já naquele país as prevalências de hipertensão, dislipidemia e diabetes tipo 2 foram respectivamente $12,2 \%, 17,4 \%$ e $1,7 \%$. No nosso estudo verificamos que $19,7 \%$ e $3,6 \%$ dos BM apresentavam hipertensão e diabetes, respectivamente. Já a prevalência de hipercolesterolemia entre os homens no nosso meio foi bem mais elevada.

Estudo avaliando apenas a saúde de mulheres bombeiras no Canadá mostrou prevalência de obesidade de $12 \%$, ao passo que no nosso estudo verificamos prevalência de $8 \%$ (TRUDEAU et al, 2018). No trabalho canadense observou-se ainda prevalência de $5 \%$ de hipertensão e de $3 \%$ de diabetes entre as mulheres, e no nosso meio verificamos prevalência de $5,2 \%$ e $2,6 \%$ destes agravos, respectivamente. Os valores encontrados no nosso meio são bastante semelhantes aos relatados na literatura. Os pesquisadores no Canadá apontaram que estas militares possuem risco cardiovascular 
moderado a alto, e propõem o desenvolvimento de programas de promoção da saúde (TRUDEAU et al, 2018).

Em todo o mundo há enorme preocupação com as doenças cardiovasculares entre bombeiros militares, bem como o impacto e custos destas no sistema de saúde (BAUR et al, 2012).

Obesidade entre bombeiros nos EUA chegou a níveis epidêmicos, motivando a criação do programa "Wellness Fitness Initiative" (WFI) desenvolvido pela Associação Internacional de Bombeiros, entre outras entidades, com o objetivo de melhorar a qualidade de vida dos Bombeiros e mostrar a importância de investir no bem-estar dos profissionais, mantendo-os saudáveis, em forma, e capazes de pronta resposta (WILKINSON et al, 2014 e POSTON et al, 2013). Portanto, prioriza-se o investimento em programas voltados a estimular a boa forma e avaliações médicas. Nas unidades que aderiram a este programa, observou-se menores índices de obesidade, hipertensão e tabagismo e maior prática de atividades físicas. Além disso, nestas unidades os bombeiros se declararam menos ansiosos e mais satisfeitos com o trabalho e com os colegas, quando comparados a bombeiros que atuam em unidades que não aderiram ao programa. O estudo mostrou, portanto, a eficácia do desenvolvimento de programas de promoção à saúde voltados para o bombeiros. Tais programas objetivam ainda reduzir os custos com recuperação da saúde dos BM, que são muito elevados (POSTON, 2013).

No nosso estudo observamos ainda altos índices de obesidade, sobrepreso e dislipidemia. Os resultados mostram que BM do sexo masculino apresentam maiores índices de hipertensão, diabetes, hipercolesterolemia, sobrepeso e obesidade quando comparados às BM do sexo feminino. Os índices de tabagismo são também maiores entre os homens. Identifica-se, portanto, esta subpopulação como mais vulnerável, e como principal alvo de estratégias para atividades e programas de prevenção.

\section{CONCLUSÕES}

Neste trabalho ficou clara a importância da identificação de fatores de risco para doenças cardiovasculares, assim como do subgrupo de bombeiros que necessitam maior 


\section{Revista FLAMMAE}

Revista Científica do Corpo de Bombeiros Militar de Pernambuco

XVIII Seminário Nacional de Bombeiros - Foz do Iguaçu PR

Vol.04 No11 - Edição Especial XVIII SENABOM - ISSN 2359-4829

Versão on-line disponível em: http://www.revistaflammae.com.

atenção. Como observado em outros países, a obesidade e hipercolesterolemia são fatores altamente prevalentes entre os $\mathrm{BM}$, merecendo atenção e intervenção imediatas. O sedentarismo é também fator de risco modificável para DCV e que merece abordagem na Corporação. O presente estudo, portanto, subsidiará a implantação de programas de promoção da saúde voltados à população de bombeiros contemplando a prática de atividades físicas e orientações nutricionais. Além da implantação de tais programas, o monitoramento, planejamento de ações, intervenções e reavaliações sistemáticas constituem os eixos de atuação da gestão do Sistema de Saúde da Corporação.

\section{REFERENCIAL BIBLIOGRÁFICO}

ASSOCIAÇÃO BRASILEIRA PARA O ESTUDO DA OBESIDADE E DA SÍNDROME METABÓLICA - Diretrizes brasileiras de obesidade 2016 / ABESO 4.ed. - São Paulo, 2016.

BARBOSA, Robson Ourives e SILVA, Eveline Fronza. Prevalência de Fatores de Risco Cardiovascular em Policiais Militares. Rev Bras Cardiol, v. 26, n. 1, p. 45-53, jan/fev, 2013.

BAUR, DOROTHEE ET AL. WEIGHT- PERCEPTION IN MALE CAREER FIREFIGHTERS AND ITS ASSOCIATION WITH CARDIOVASCULAR RISK FACTORS. BMC PUBLIC HEALTH, V. 25, N. 12, P. 480, JUN, 2012.

CALAMITA, Zamir et al. Fatores de risco para doenças cardiovasculares no policial militar . Revista Bras. Med. Trab, São Paulo, v. 8, n.1 , jan, 2010.

DE OLIVEIRA, José Egidio Paulo; Vencio, Sérgio. Diretrizes da Sociedade Brasileira de Diabetes: 2014-2015/Sociedade Brasileira de Diabetes, São Paulo, 2015.

KALES, Stefanos et al. Emergency duties and deaths from heart disease among firefighters in the United States. N Engl J Med , v. 356, p. 1207-1215, mar, 2007. 
Revista Científica do Corpo de Bombeiros Militar de Pernambuco

XVIII Seminário Nacional de Bombeiros - Foz do Iguaçu PR

Vol.04 No11 - Edição Especial XVIII SENABOM - ISSN 2359-4829

Versão on-line disponível em: http://www.revistaflammae.com.

KISS, P ET AL. CARDIORESPIRATORY FITNESS IN A REPRESENTATIVE SAMPLE OF BELGIAN FIREFIGHTERS. OCCUPATIONAL MEDICINE, V. 64, N. 8, P. 589-594, DEZ, 2014.

LI, Kaigang et al. Cardiac health and fitness of Colorado male/female firefighters. Occup Med (Lond), v. 67, n. 4, p. 268-273, jun, 2017.

LI, Kaigang et al. Correlates of atherosclerotic cardiovascular disease risk in older Colorado firefighters. Occupational Medicine, v. 68, n. 1. p. 51-55, jan, 2018

MENDIS, Shanthi; Puska, Pekka e Norrving, Bo. Global Atlas on Cardiovascular Disease Prevention and Control. World Health Organization, Geneva 2011.

MINAYO, Maria Cecília de Souza et al. Impacto das atividades profissionais na saúde física e mental dos policiais civis e militares do Rio de Janeiro (RJ, Brasil). Ciência \& Saúde Coletiva, v. 16, n. 4, p. 2199-2209, 2011.

NOGUEIRA, Eugênio et al. Body composition is strongly associated with cardiorespiratory fitness in a large Brazilian military firefighter cohort: The Brazilian Firefighters Study. J Strength Cond Res, v. 30, n. 1, p. 33-38, jan, 2016.

OLIVEIRA, Eduardo de Almeida Magalhaes e Anjos, Luiz Antonio. Medidas antropométricas segundo aptidão cardiorrespiratória em militares da ativa, Brasil. Rev Saúde Pública, v. 42, n. 2, p. 217-23, 2008.

PEDERSEN, Julie Elbaek et al. Incidence of cardiovascular disease in a historical cohort of Danish firefighters. Occup Environ Med. v. 75, n. 5, p. 337-343, mai, 2018.

POSTON, Walker et al. An examination of the benefits of health promotion programs for the national fire service. BMC Public Health, v. 13, n. 805, Set, 2013.

POSTON, Walker et al. The prevalence of overweight, obesity, and substandard fitness in a population-based firefighter cohort. J Occup Environ Med, v. 53, n. 3, p. 266-73, Mar, 2011.

REZENDE, Fabiane Aparecida Canaan et al. Índice de Massa Corporal e Circunferência Abdominal: Associação com Fatores de risco Cardiovascular. Arq Bras Cardiol, v. 87, n. 6, p. 728-734, jan, 2006. 
Revista Científica do Corpo de Bombeiros Militar de Pernambuco XVIII Seminário Nacional de Bombeiros - Foz do Iguaçu PR Vol.04 No11 - Edição Especial XVIII SENABOM - ISSN 2359-4829

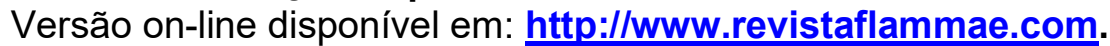

RODRIGUES, Liraine et al. Estudo da Prevalência dos Critérios Clínicos para a Síndrome Metabólica em Bombeiros Militares de Um Batalhão da Região Centro-Sul de Belo Horizonte. e-Scientia, Belo Horizonte, v. 5, n. 1, p. 31-38, jul, 2012.

SCHMIDT, Maria I., DUNCAN, Bruce Bartholow et al. CHRONIC NONCOMMUNICABLE DISEASES IN BRAZIL: BURDEN AND CURRENT CHALLENGES. LANCET, V. 4, N. 377(9781), P. 1949-61, MAI, 2011.

SMITH, DENISE ET AL. THE PREVALENCE OF CARDIOVASCULAR DISEASE RISK FACTORS AND OBESITY IN FIREFIGHTERS. JOURNAL OF OBESITY, V. 2012, JUL, 2012.

SOCIEDADE BRASILEIRA DE HIPERTENSÃO. Diretrizes Brasileiras de Hipertensão VI. Revista Hipertensão, v. 13, n. 1, p. , Jan/março, 2010.

SOTERIADES, Elpidoforos et al. Cardiovascular disease in US firefighters. A systematic review. Cardiol Rev , v. 19, p. 202-215, jul-ago 2011.

SOTERIADES, Elpidoforos et al. Obesity and Cardiovascular Disease Risk Factors in Firefighters: A Prospective Cohort Study. Obesity Research, v. 13, n. 10, p. 1756-63, out, 2005.

TRUDEAU, François et al. Cardiovascular Disease Risk Factors in Québec Male Firefighters. J Occup Environ Med, v. 60, n. 6, jun, 2018.

TRUDEAU, François et al. Cardiovascular disease risk in female firefighters. Occup Med (Lond), Mai, 2018.

WILKINSON, Michelle Lynn et al. Physician weight recommendations for overweight and obese fireighters, United States, 2011-2012. Prev Chronic Disease, v. 11, E116, jul, 2014.

XAVIER, Hermes Toros et al. Sociedade Brasileira de Cardiologia - V Diretriz Brasileira de Dislipidemias e Prevenção da Aterosclerose. Arq Bras Cardiol v. 101, n. 4 , Supl. 1, p. 1-22, out, 2013. 